\title{
Complex investigations of phase diagram of Ni-Pt-Mn-Ga Heusler alloys
}

\author{
M. A. Zagrebin ${ }^{1,2, \dagger}$, S. A. Derevyanko ${ }^{1}$, V. V. Sokolovskiy ${ }^{1,3}$, V. D. Buchelnikov ${ }^{1}$ \\ †miczag@mail.ru
}

\author{
${ }^{1}$ Chelyabinsk State University, 129, Bratiev Kashirinykh str., Chelyabinsk, 454001, Russia \\ ${ }^{2}$ National Research South Ural State University, 76, Lenin prospekt, Chelyabinsk, 454080, Russia \\ ${ }^{3}$ National University of Science and Technology "MIS\&S”, 4, Leninskiy prospect, Moscow, 119991, Russia
}

In this paper, structural (lattice parameter, tetragonality distortions) and magnetic (total magnetic moments, magnetic exchange parameters) properties for $\mathrm{Ni}_{2-x} \mathrm{Pt}_{x} \mathrm{MnGa}$ shape memory Heusler alloys were studied using density functional theory. It is shown that the equilibrium crystal lattice parameter of the austenite increases with increasing Pt content. The largest tetragonal distortions are observed in the $\mathrm{Ni}_{1.0} \mathrm{Pt}_{1.0} \mathrm{MnGa}$ alloy, in which the $c / a$ ratio reaches 1.32. An investigation of the magnetic exchange parameters shows that the largest contribution to the total exchange energy is associated with interaction between nearest neighboring $\mathrm{Ni}-\mathrm{Mn}$ atoms. In the martensitic state, the magnetic exchange parameters increase. The concentration dependences of the Curie temperature were calculated by means Monte Carlo method and mean field approximation. It is shown that the Curie temperatures of the austenite calculated using the Monte Carlo simulation and the mean field approximation have similar values, while the Curie temperature in martensite obtained within the mean field approximation is higher than the Curie temperature obtained from the Monte Carlo simulation. The temperatures of the martensitic phase transitions are estimated. It is shown that the temperature of martensitic phase transition increases with increasing of Pt content. Based on these temperatures, a phase diagram for $\mathrm{Ni}_{2-x} \mathrm{Pt}_{x} \mathrm{MnGa}$ Heusler alloys For the whole range of Pt concentration $(0 \leq x \leq 2)$ is constructed. Comparison of the obtained values and experimental results was conducted. It is shown that the calculated Curie temperatures are in good agreement with the experimental data.

Keywords: density functional theory, exchange interaction, Curie temperature, Heusler alloys.

УДК 538.9

\section{Комплексное исследование фазовой диаграммы сплавов Гейслера Ni-Pt-Mn-Ga}

\author{
Загребин М.А. ${ }^{1,2 \dagger}$, Деревянко С. А. ${ }^{1}$, Соколовский В. В. ${ }^{1,3}$, Бучельников В. Д. ${ }^{1}$ \\ 1Челябинский государственный университет, ул. Братьев Кашириных, 129, Челябинск, 454001, Россия \\ ${ }^{2}$ Южно-Уральский государственный университет, просп. Ленина, 76, Челябинск, 454080, Россия
}

${ }^{3}$ Национальный исследовательский технологический университет «МИСиС», Ленинский пр. 4, Москва, 119049, Россия

В данной работе с помощью теории функционала плотности вычислены структурные (параметр кристаллической решетки, тетрагональные искажения) и магнитные (полные магнитные моменты, параметры магнитного обменного взаимодействия) свойства для сплавов Гейслера $\mathrm{Ni}_{2-x} \mathrm{Pt}_{x} \mathrm{MnGa}$ с эффектом памяти формы. Показано, что равновесный параметр кристаллической решетки аустенита увеличивается с увеличением содержания Рt. Наибольшие тетрагональные искажения наблюдаются в сплаве $\mathrm{Ni}_{1,0} \mathrm{Pt}_{1,0} \mathrm{MnGa}$, в котором соотношение кристаллографических осей c/a достигает 1,32. Исследование параметров магнитного обменного взаимодействия показывает, что наибольший вклад в полную энергию обменного взаимодействия связан с взаимодействием между парой ближайших соседних атомов Ni-Mn. В мартенситной фазе параметры магнитного обменного взаимодействия увеличиваются. Вычислены концентрационные зависимости температуры Кюри с помощью метода Монте-Карло и приближения среднего поля. Показано, что температуры Кюри аустенита, вычисленные с помощью метода Монте-Карло и приближения среднего поля имеют близкие значения, в то время как температуры Кюри мартенсита, полученные в рамках приближения среднего поля выше чем температуры Кюри, полученные из моделирования с помощью метода Монте-Карло. Проведена оценка температуры мартенситного фазового перехода. Показано, что температура мартенситного перехода увеличивается с увеличением содержания Pt. На основании данных температур построена фазовая диаграмма для сплавов Гейслера $\mathrm{Ni}_{2-x} \mathrm{Pt}_{x} \mathrm{MnGa}$ во всем интервале концентраций $\mathrm{Pt}(0 \leq x \leq 2)$. Проведено сравнение полученных значений с экспериментальными результатами. Показано, что полученные в результате вычислений температуры Кюри находятся в хорошем согласии с экспериментальными данными.

Ключевые слова: теория функционала плотности, обменное взаимодействие, температура Кюри, сплавы Гейслера. 


\section{1. Введение}

Эффект гигантской магнитодеформации составляющей около $10 \%$, наблюдаемый в сплавах Гейслера $\mathrm{Ni}_{2} \mathrm{MnGa}$, делает его важным материалом для практического применения [1 - 3]. Помимо эффекта гигантской магнитодеформации, магнитокалорический эффект наблюдаемый в сплаве $\mathrm{Ni}_{2} \mathrm{MnGa}$ предоставляет возможность использовать его при магнитном охлаждении [4]. Однако, низкая температура мартенситного перехода $\left(T_{m} \approx 200 \mathrm{~K}\right)$ и хрупкость сплава ограничивает его практическое применение, что вызывает необходимость поиска новых ферромагнитных (ФМ) сплавов с памятью формы $[5,6]$.

Теоретические исследования, проведенные группой под руководством проф. П. Энтеля (P. Entel et al.) показали, что изоэлектронное замещение атомами $\mathrm{Pt}$ оставляет неизменным соотношение $e / a=7,5$ и приводит к появлению магнитодеформаций, достигающих около $14 \%$ в сплаве $\mathrm{Ni}_{100} \mathrm{Pt}_{100} \mathrm{MnGa}$, что делает четырехкомпонентные сплавы $\mathrm{Ni}-\mathrm{Pt}-\mathrm{Mn}-\mathrm{Ga}$ достойными кандидатами для практической реализации устройств на основе магнитного эффекта памяти формы [7]. Связано это с тем, что, несмотря на одинаковое число валентных электронов у $\mathrm{Ni}$ и Pt, их электронные конфигурации различны, так как Pt имеет на один электрон $d$-типа больше, и на один электрон $s$-типа меньше, чем $\mathrm{Ni}$ [7]. Кроме того, исследования показали, что замещение атомами Pt приводит к увеличению постоянной решетки от $a_{0}=5,81 \AA$ для фазы L2 1 сплава $\mathrm{Ni}_{2} \mathrm{MnGa}$ до 6,05 для сплава $\mathrm{Ni}_{1,0} \mathrm{Pt}_{1,0} \mathrm{MnGa}$, и $6.23 \AA$ в случае сплава $\mathrm{Pt}_{2} \mathrm{MnGa}$.

В недавней работе [8] С. Синх с соавт. (S. Singh et al.), представили результаты экспериментального исследования кристаллической структуры, магнитных свойств и фазовых переходов серии четырехкомпонентных сплавов $\mathrm{Ni}_{2-x} \mathrm{Pt} \mathrm{MnGa}(0 \leq x \leq 1)$, с помощью рентгеновской дифракции, измерения низкополевой намагниченности образцов и нейтронографии. В работе были представлены концентрационные зависимости параметров кристаллической решетки, магнитного момента и температур фазовых переходов. Было показано, замещение атомов $\mathrm{Ni}$ атомами $\mathrm{Pt}$ в $\mathrm{Ni}_{2} \mathrm{MnGa}$ приводит к увеличению температуры мартенситного перехода, при этом температура магнитного перехода уменьшается.

Таким образом, в данной работе рассматривается переход по концентрации от сплава $\mathrm{Ni}_{2} \mathrm{MnGa}$ к сплаву $\mathrm{Pt}_{2} \mathrm{MnGa}$, путем частичной замены атомов $\mathrm{Ni}$ атомами $\mathrm{Pt}$. Мы представляем результаты систематических исследований магнитных свойств сплавов Гейслера $\mathrm{Ni}_{2-x} \mathrm{Pt}_{x} \mathrm{MnGa}$ $(0 \leq x \leq 2)$ с помощью первопринципных расчетов в сочетании с моделированием Монте-Карло (МК) и теорией среднего поля.

\section{2. Детали вычислений}

Для исследования основного состояния и магнитных свойств сплавов Гейслера $\mathrm{Ni}_{2-x} \mathrm{Pt}_{x} \mathrm{MnGa}(0 \leq x \leq 2)$, были использованы метод присоединенных плоских волн и метод Корринги-Кона-Ростокера, реализованные в вычислительных пакетах VASP (Vienna ab initio simulation program) $[9,10]$ и SPR-KKR (Spin-Polarized-Relativistic Korringa-Kohn-Rostoker code) [11], соответственно. Для учета обменно-корреляционного взаимодействия было использовано приближение обобщенного градиента (General gradient approximation - GGA) в параметризации Пердью-Бурке-Эрнзерхофа (Perdew-BurkeErnzerhof - PBE) [12]. Геометрическая оптимизация структуры аустенитной фазы была проведена с использованием пакета VASP в сочетании с подходом суперячейки, состоящей из 16 атомов. Для оптимизации была использована структура L2 (пространственная группа симметрии $F m \overline{3} m$, № 225). Для сплава с формулой $X_{2} Y Z$ она состоит из четырех взаимопроникающих ГЦК подрешеток: двух подрешеток из атомов $X$, которые расположены на позиции Вайкоффа $8 c$ (позиции $(1 / 4,1 / 4,1 / 4)$ и $(3 / 4,3 / 4,3 / 4))$, и подрешеток из атомов $Z$ и $Y$, которые занимают позиции $4 a$ и $4 b((0,0,0)$ и $(1 / 2,1 / 2,1 / 2))$, соответственно. Все вычисления проводились для ФМ конфигурации, в которой все атомы имеют положительные магнитные моменты.

Оптимизированные параметры решетки далее были использованы для расчета магнитных моментов и параметров магнитного обменного взаимодействия $\left(J_{i j}\right)$ при помощи пакета SPR-KKR. Магнитные моменты, параметры магнитного обменного взаимодействия были рассчитаны с использованием спин-поляризованного скалярно-релятивистского гамильтониана (SP-SREL) Дирака с использованием параметризации GGA-PBE.

Для расширения результатов первопринципных вычислений, полученных при нулевой температуре до конечных температур было проведено МК-моделирование, с использованием классической трехмерной модели Гейзенберга $\left(H=-\sum_{i j} J_{i j} \mathbf{S}_{i} \mathbf{S}_{j}\right)$ в нулевом магнитном поле. В этом случае, рассчитанные параметры магнитного обменного взаимодействия $\left(J_{i j}\right)$ и магнитные моменты элементов были взяты в качестве входных параметров модели. МК-моделирование проводилось для реальной ячейки, состоящей из 3925 атомов и периодических граничных условий, с использованием алгоритма Метрополиса [13]. Для стехиометрического сплава $\mathrm{Ni}_{2} \mathrm{MnGa}$ модельная решетка содержит 1098 атомов Mn, 1099 Ga и 1728 атомовNi.

\section{3. Результаты и обсуждение}

Первым шагом наших расчетов было определение равновесных параметров решетки сплавов Гейслера $\mathrm{Ni}_{2-x} \mathrm{Pt}_{x} \mathrm{MnGa}(0 \leq x \leq 2)$ путем вычисления зависимости полной энергии сплава от параметра решетки $a$. Исследования показывают, что равновесные параметры кристаллической решетки $a_{0}$ аустенита увеличиваются с увеличением концентрации Pt. Данное обстоятельство вызвано тем фактом, что атомный радиус Pt больше атомного радиуса $\mathrm{Ni}$. Для Стехиометрического сплава $\mathrm{Ni}_{2} \mathrm{MnGa} a_{0}=5,812 \AA$, для сплава $\mathrm{Pt}_{2} \mathrm{MnGa} a_{0}=6,230 \AA$. Полученные в результате геометрической оптимизации равновесные параметры решетки рассматриваемых сплавов находятся в хорошем согласии с опубликованными экспериментальными и теоретическими значениями $[8,14]$. 
Для того чтобы исследовать возможность мартенситного превращения в этих сплавах, были проведены вычисления полной энергии в зависимости от тетрагональных искажений кубической структуры вдоль оси $z$. Хорошо известно, что переход аустенит-мартенсит требует, чтобы мартенситная фаза обладала меньшей энергией по сравнению с аустенитной, при неизменном объеме. Проведенные расчеты показывают, что в случае $\mathrm{Ni}_{2} \mathrm{MnGa}$ в мартенситной фазе реализуется отношение $c / a$, равное 1,26 . Увеличение концентрации Pt приводит сначала к увеличению отношения $c / a$. Максимальное значение $c / a$ достигается для сплава $\mathrm{Ni}_{1,0} \mathrm{Pt}_{1,0} \mathrm{MnGa}$ и составляет 1,32. Для сплава $\mathrm{Ni}_{0.75} \mathrm{Pt}_{1.25} \mathrm{MnGa}$ степень тетрагональности уменьшается до значения $c / a=1,30$ и не изменяется с дальнейшим увеличением концентрации Pt. Для сплава $\mathrm{Pt}_{2} \mathrm{MnGa}$ устойчивое мартенситное состояние имеет место при $c / a=1,30$. Также было отмечено, что для серии сплавов $\mathrm{Ni}_{2-x} \mathrm{Pt}_{x} \mathrm{MnGa}$ разность энергий между аустенитом и мартенситом возрастает с увеличением содержания Pt. Это важно, так как это различие является количественным показателем температуры мартенситного превращения $\left(T_{m}\right)$ в соответствии с отношением $\Delta E \approx k_{B} T_{m}$. Здесь $k_{B}-$ постоянная Больцмана [7].
В таблице 1 приведены вычисленные равновесные параметры решетки, степень тетрагональных искажений $c / a$, полная энергия $\Delta E$ относительно равновесной энергий всех сплавов $\mathrm{Ni}_{2-x} \mathrm{Pt}_{x} \mathrm{MnGa}(0 \leq x \leq 2)$.

Дальнейшее исследование сплавов Гейслера $\mathrm{Ni}_{2-x} \mathrm{Pt}_{x} \mathrm{MnGa}(0 \leq x \leq 2)$ связано c ab initio расчетами констант магнитного обменного взаимодействия для аустенитного и мартенситного состояний. Эти расчеты были выполнены для ячейки, состоящей из четырех атомов с использованием приближения когерентного потенциала (Coherent potential approximation - CРA), реализованного в пакете SPR-KKR.

На рис. 1 представлены постоянные магнитного обменного взаимодействия сплавов $\mathrm{Ni}_{2-x} \mathrm{Pt}_{x} \mathrm{MnGa}$ в зависимости от расстояния между взаимодействующими парами атомов для аустенитной (рис. $1 \mathrm{a}, 1 \mathrm{c}, 1 \mathrm{e}$ ) и мартенситной (рис. $1 \mathrm{~b}, 1 \mathrm{~d}, 1 \mathrm{f})$ ) фаз. На рис. 1 положительные значения обменных постоянных $\left(J_{i j}>0\right)$ указывают на ФМ взаимодействие, тогда как отрицательные значения $\left(J_{i j}<0\right)-$ на антиферромагнитное $(\mathrm{AФМ)} \mathrm{взаи-}$ модействие. Отметим, что обменные взаимодействия с участием атомов $\mathrm{Ga}$ и $\mathrm{Pt}$ (за исключением $\mathrm{Mn}-\mathrm{Pt}$ ) очень малы и на рисунках не представлены. Из рис. 1 видно,

Табл. 1. Вычисленные равновесные постоянные кристаллической решетки $a_{0}$ (в $\AA$ ), степень тетрагональных искажений (c/a) и полная энергия системы относительно равновесной системы в аустенитном состоянии $\Delta E$ (в мэВ/атом) для всех рассматриваемых сплавов $\mathrm{Ni}_{2-x} \mathrm{Pt}_{x} \mathrm{MnGa}$.

Table 1. Calculated equilibrium lattice parameters $a_{0}$ (in $\AA$ ), tetragonality $(c / a)$ and the total energy difference $\Delta E$, relative to equilibrium energies (in $\mathrm{meV} /$ atom) for all considered $\mathrm{Ni}_{2-x} \mathrm{Pt}_{x} \mathrm{MnGa}$ alloys.

\begin{tabular}{|c|c|c|c|c|c|c|c|c|c|}
\hline$x$ & 0 & 0,25 & 0,50 & 0,75 & 1,0 & 1,25 & 1,5 & 1,75 & 2,0 \\
\hline$a_{0}$ & 5,812 & 5,885 & 5,948 & 6,002 & 6,047 & 6,101 & 6,149 & 6,192 & 6,230 \\
\hline$c / a$ & 1,26 & 1,29 & 1,30 & 1,31 & 1,32 & 1,30 & 1,30 & 1,30 & 1,30 \\
\hline$\Delta E$ & 7,258 & 18,250 & 27,633 & 33,533 & 39,009 & 47,176 & 53,289 & 58,689 & 63,012 \\
\hline
\end{tabular}
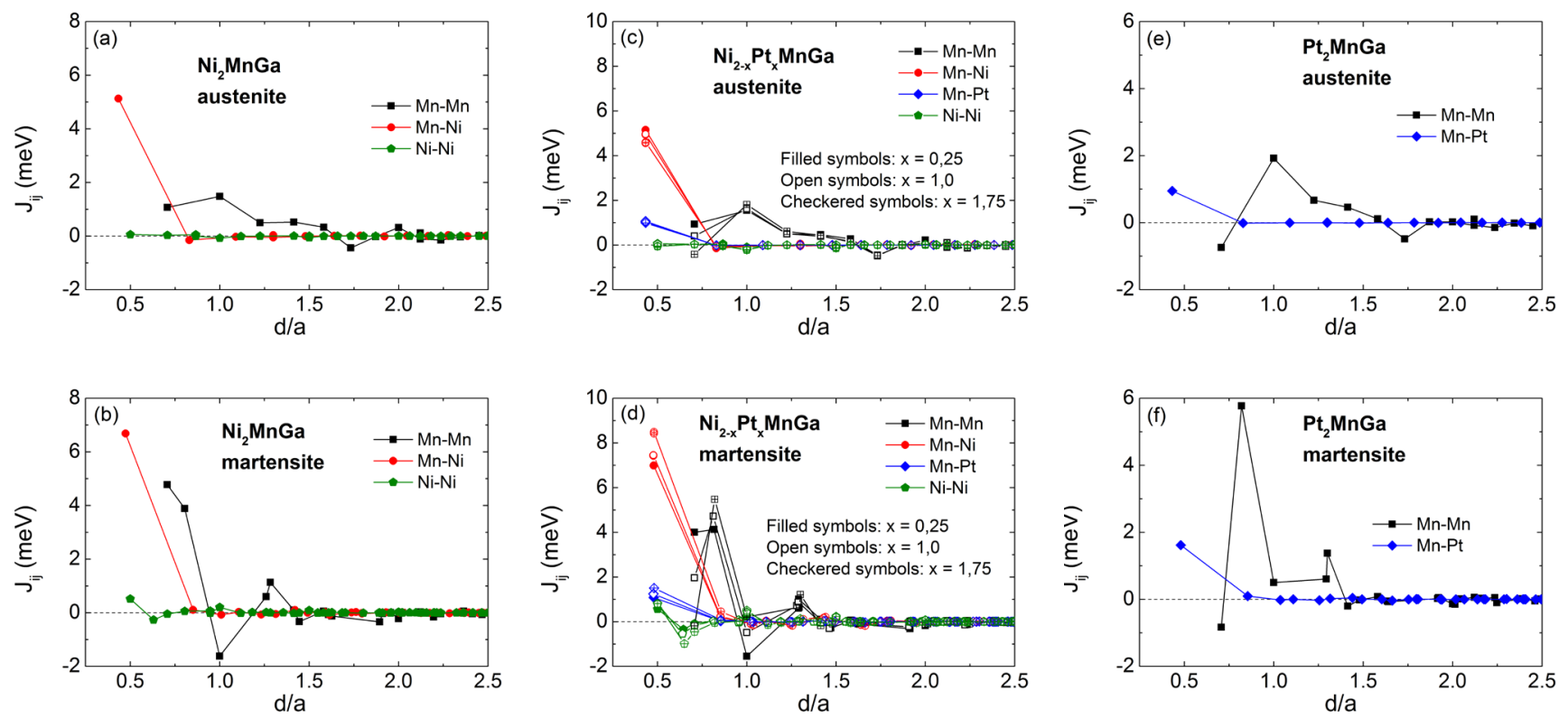

Рис. 1. Постоянные магнитного обменного взаимодействия в зависимости от расстояния $(d / a)$ между атомами $i$ и $j$ для сплавов $\mathrm{Ni}_{2} \mathrm{MnGa}(\mathrm{a}, \mathrm{b}) ; \mathrm{Ni}_{2-x} \mathrm{Pt}_{x} \mathrm{MnGa}(x=0,25 ; 1,0 ; 1,75)$ (c, d); $\mathrm{Pt}_{2} \mathrm{MnGa}(\mathrm{e}, \mathrm{f})$ - в аустенитном (a, c, e) и мартенситном (b, d, f) состояниях.

Fig. 1. Exchange coupling constants as a function of distance $(d / a)$ between atoms $i$ and $j$ for $\mathrm{Ni}_{2} \mathrm{MnGa}(\mathrm{a}, \mathrm{b}) ; \mathrm{Ni}_{2-x} \mathrm{Pt}_{x} \mathrm{MnGa}(x=0,25 ; 1,0 ; 1,75)$ (c, d); $\mathrm{Pt}_{2} \mathrm{MnGa}(\mathrm{e}, \mathrm{f})$ alloys in the austenitic (a, c, e) and martensitic (b, d, f) states. 
что постоянные магнитного обменного взаимодействия для всех сплавов демонстрируют колебательное затухающее поведение.

Магнитные взаимодействия между $\mathrm{Mn}-\mathrm{Ni}, \mathrm{Mn}-\mathrm{Pt}$ являются ФМ и резко уменьшаются при увеличении расстояния между атомами, в свою очередь взаимодействия $\mathrm{Mn}-\mathrm{Mn}$ также являются преимущественно ФМ, однако могут иметь и АФМ вклад в зависимости от расстояния между атомами Mn. Из рис. 1 видно, что межподрешеточные взаимодействия между ближайшими парами атомов $\mathrm{Ni}(\mathrm{Pt})-\mathrm{Mn}$ обеспечивают наибольший вклад в обмен по сравнению с внутриподрешеточными взаимодействиями ( $\mathrm{Ni}-\mathrm{Ni}$ и $\mathrm{Mn}-\mathrm{Mn})$. Такое сильное межподрешеточное ФМ взаимодействие может быть связано с более коротким расстоянием $\mathrm{Ni}-\mathrm{Mn}\left(d=\sqrt{3} / 4 a_{0}\right)$ по сравнению с внутриподрешеточными Ni-Ni и $\mathrm{Mn}-\mathrm{Mn}$ расстояниями $\left(d=1 / 2 a_{0}\right.$ и $\left.d=\sqrt{2} / 2 a_{0}\right)$ соответственно. Величина взаимодействия $\mathrm{Mn}-\mathrm{Ni}$ между ближайшими соседями с увеличением концентрации $\mathrm{Pt}$ уменьшается с 5 мэВ (для $x=0,25)$ до 4,5 мэВ (для $x=1,75$ ). Что касается взаимодействия $\mathrm{Mn}-\mathrm{Mn}$ в первой координационной сфере, то для сплава $\mathrm{Ni}_{1,75} \mathrm{Pt}_{0,25} \mathrm{MnGa}$ данное взаимодействие является ФМ, и с увеличением концентрации Pt величина взаимодействия уменьшается. Для сплава $\mathrm{Ni}_{0,25} \mathrm{Pt}_{1,75} \mathrm{MnGa}$ взаимодействие $\mathrm{Mn}-\mathrm{Mn}$ между ближайшими соседями становится слабым АФМ. Взаимодействия $\mathrm{Ni}-\mathrm{Ni}$ и $\mathrm{Mn}-\mathrm{Pt}$ имеют слабую зависимость от концентрации Pt.

В мартенситной фазе (рис. $1 \mathrm{~b}, 1 \mathrm{~d}, 1 \mathrm{f}$ ) величина всех взаимодействий между ближайшими атомами увеличивается примерно в 1,5-2 раза в сравнении с аустенитной фазой. Характер зависимостей величины взаимодействий от концентрации Pt в сплаве аналогичный случаю аустенитного состояния.

Используя полученные постоянные магнитного обменного взаимодействия, можно оценить температуру Кюри с помощью хорошо зарекомендовавшей модели Гейзенберга в рамках приближения среднего поля [15]

$$
\left\langle s^{\mu}\right\rangle=\frac{2}{3 k_{B} T} \sum_{v} J_{0}^{\mu v}\left\langle s^{v}\right\rangle,
$$

где $J_{0}^{\mu v}=\sum_{i, j} J_{i j}$ - эффективные параметры обменного взаимодействия; $\mu, v-$ различные подрешетки; $\left\langle s^{v}\right\rangle-$ среднее значение $z$-составляющей спина атома, расположенная в подрешетке $v$. Уравнение (1) имеет нетривиальные решения, если определитель равен нулю. В этом случае наибольшее собственное значение дает температуру Кюри [15].
Полученные значения температуры Кюри для аустенитного $T_{C}{ }^{A}$ и мартенситного $T_{C}{ }^{M}$ состояний вместе с полными магнитными моментами приведены в таблице 2.

Заключительный этап настоящего исследования связан с расчетами температурных кривых намагниченности при конечных температурах для сплавов $\mathrm{Ni}_{2-x} \mathrm{Pt}$ MnGa $(0 \leq x \leq 2)$ с помощью МК-моделирования. Для выполнения этого моделирования была использована классическая модель Гейзенберга с дальнодействующими обменными взаимодействиями, взятыми из $a b$ initio расчетов в качестве входных данных отдельно для аустенитного и мартенситного состояний. В результате для исследуемых сплавов был получен набор кривых намагниченности аустенита и мартенсита в зависимости от температуры. Ввиду того, что во всех случаях наблюдалось сходное поведение фазового перехода ФМ-парамагнетик, пропустим подробное обсуждение данных кривых, и вместо этого перейдем к обсуждению $T-x$ фазовых диаграмм сплавов $\mathrm{Ni}_{2-x} \mathrm{Pt}_{x} \mathrm{MnGa}(0 \leq x \leq 2)$, показанных на рис. 2. Для оценки температуры Кюри была построена функция $m(T)=M^{1 / \beta}(T)$, которая линейно убывает с ростом температуры. Температуру Кюри можно оценить на пересечении $m$ с осью Т. Здесь $M-$ полная намагниченность, а $\beta$ - критический индекс, который в случае трехмерной модели Гейзенберга равен 0,3646. Что касается температуры мартенситного превращения, то она была оценена с помощью грубого приближения из отношения $\left(\Delta E \approx k_{B} T_{m}\right)$, упомянутого выше.

Чтобы сравнить полученные результаты с экспериментом, на рис. 2а представлены результаты МК-вычислений температур Кюри $\left(T_{C}\right)$, оценки температур мартенситного фазового перехода $\left(T_{m}\right)$ для сплавов $\mathrm{Ni}_{2-x} \mathrm{Pt}_{x} \mathrm{MnGa}(0 \leq x \leq 1)$ в зависимости от концентрации $\mathrm{Pt}(x)$ и экспериментальные данные. Стоит отметить, что реализация уравнения $\left(\Delta E \approx k_{B} T_{m}\right)$ приводит к более низкой величине температуры мартенситного перехода $(84,2 \mathrm{~K})$ для $\mathrm{Ni}_{2} \mathrm{MnGa}$ по сравнению с экспериментальной [14]. Чтобы правильно описать мартенситные фазовые переходы, происходящие в этих сплавах, следует учитывать магнитные возбуждения. Однако расчетные температуры могут позволить нам качественно описать увеличение температуры мартенситного перехода, обнаруженное экспериментально [8].

Значения температуры Кюри в аустенитном состоянии $\left(T_{C}{ }^{A}\right)$, полученные с помощью МК-моделирования находятся в хорошем согласии с экспериментальными значениями из работы [8].

Табл. 2. Вычисленные с помощью приближения среднего поля температуры Кюри аустенита $T_{C}{ }^{A}$ и мартенсита $T_{C}{ }^{M}$ (в К) вместе с полными магнитными моментами (в $\mu_{B}$ ) для всех рассматриваемых сплавов $\mathrm{Ni}_{2-x} \mathrm{Pt}_{x} \mathrm{MnGa}$.

Table 2. Calculated by means mean field approximation Curie temperatures in austenitic $T_{C}^{{ }^{x}}$ and martensitic $T_{C}^{M}$ states (in K) together with total magnetic moments $\left(\right.$ in $\mu_{B}$ ) for all considered $\mathrm{Ni}_{2-x} \mathrm{Pt}_{x} \mathrm{MnGa}$ alloys.

\begin{tabular}{|c|c|c|c|c|c|c|c|c|c|}
\hline$x$ & 0 & 0,25 & 0,50 & 0,75 & 1,0 & 1,25 & 1,5 & 1,75 & 2,0 \\
\hline$T_{C}{ }^{A}$ & 408,2 & 392,1 & 372,2 & 354,0 & 335,0 & 317,3 & 283,4 & 280,3 & 146,3 \\
\hline$\mu^{A}$ & 4,015 & 4,078 & 4,128 & 4,155 & 4,172 & 4,174 & 4,170 & 4,156 & 4,246 \\
\hline$T_{C}{ }^{M}$ & 562,5 & 580,7 & 583,7 & 590,6 & 590,4 & 613,2 & 639,0 & 663,4 & 436,9 \\
\hline$\mu^{M}$ & 4,157 & 4,188 & 4,204 & 4,211 & 4,211 & 4,208 & 4,209 & 4,205 & 4,228 \\
\hline
\end{tabular}



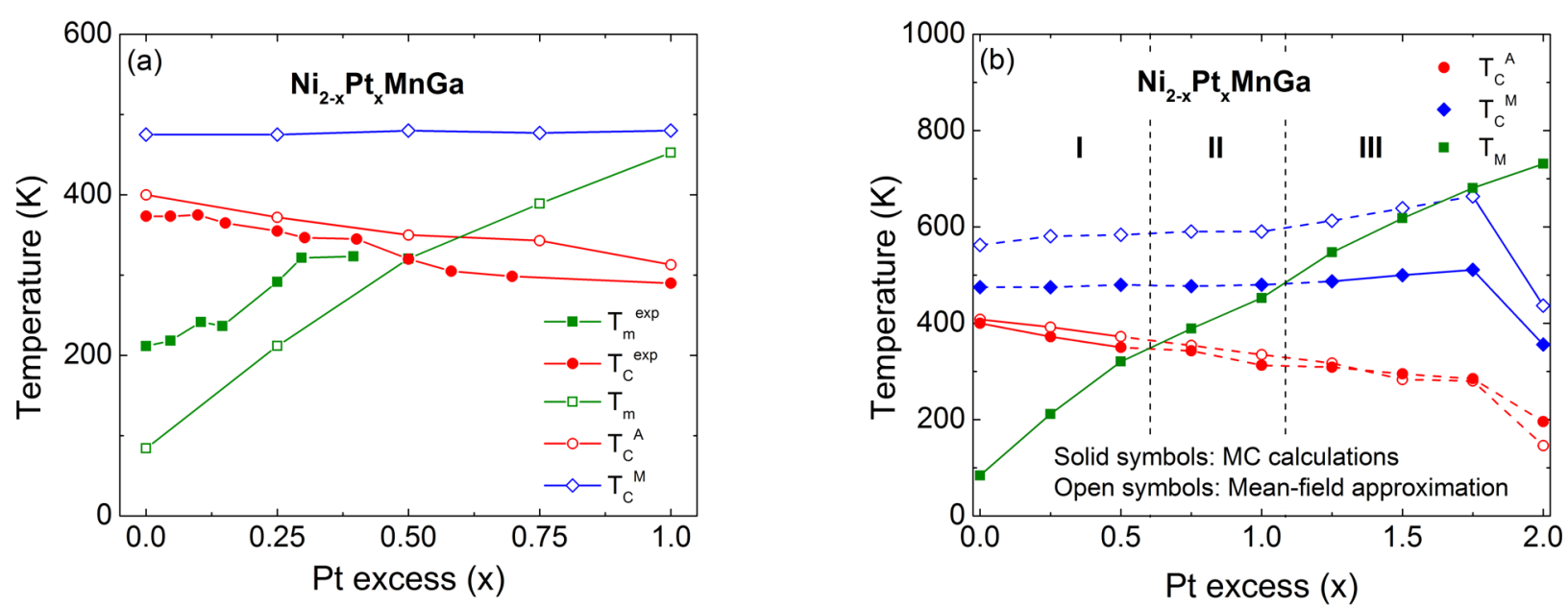

Pис. 2. Температуры Кюри $\left(T_{C}\right)$, полученные с помощью Монте-Карло моделирования и оцененные температуры мартенситного фазового перехода $\left(T_{m}\right)$ для сплавов $\mathrm{Ni}_{2-x} \mathrm{Pt}_{x} \mathrm{MnGa}(0 \leq x \leq 1)$ как функции концентрации $\mathrm{Pt}(x)$ в сравнении с экспериментальными данными, взятыми из [8] (а); Температуры Кюри $\left(T_{c}\right)$, вычисленные с помощью Монте-Карло моделирования и оцененные температуры мартенситного фазового перехода $\left(T_{m}\right)$ для сплавов $\mathrm{Ni}_{2-x} \mathrm{Pt}_{x} \mathrm{MnGa}(0 \leq x \leq 2)$ как функции концентрации Pt $(x)$ в сравнении с температурами Кюри, оцененными с помощью приближения среднего поля (b).

Fig. 2. Monte Carlo results of Curie temperatures $\left(T_{C}\right)$ and estimated temperatures of the martensitic phase transition $\left(T_{m}\right)$ for $\mathrm{Ni}_{2-x} \mathrm{Pt} \mathrm{MnGa}_{x}$ $(0 \leq x \leq 1)$ alloys as functions of Pt concentration $(x)$ in comparison with experimental data taken from [8] (a); Monte Carlo results of Curie temperatures $\left(T_{C}\right)$ and estimated temperatures of the martensitic phase transition $\left(T_{m}\right)$ for $\mathrm{Ni}_{2-x} \mathrm{Pt}_{x} \mathrm{MnGa}(0 \leq x \leq 2)$ alloys as functions of $\mathrm{Pt}$ concentration $(x)$ in comparison with Curie temperatures estimated by using mean field approximation (b).

Далее обсудим $T-x$ фазовые диаграммы для сплавов $\mathrm{Ni}_{2-x} \mathrm{Pt} \mathrm{MnGa}$, построенные в широком интервале концентрации $\mathrm{Pt}$ (рис. 2b). На рис. 2b представлены результаты МК-моделирования температур Кюри $\left(T_{C}\right)$, оценки температур мартенситного фазового перехода $\left(T_{m}\right)$ для сплавов $\mathrm{Ni}_{2-x} \mathrm{Pt}_{x} \mathrm{MnGa}(0 \leq x \leq 2)$ в зависимости от концентрации $\mathrm{Pt}(x)$ и значения температур Кюри, полученные с помощью приближения среднего поля из таблицы 2. Здесь сплошными линиями с символами изображены реальные температуры Кюри, в то время как штриховыми линиями с символами - «виртуальные» температуры. Здесь «виртуальная» температура Кюри ответственна за гипотетический магнитный переход при гораздо более высоких температурах, чем температура мартенситного превращения [16].

Следует отметить, что температуры Кюри, полученные из МК-моделирования и приближения среднего поля имеют одинаковый характер поведения с увеличением концентрации Pt. Температуры Кюри аустенита $\left(T_{C}^{A}\right)$ имеют близкие значения, в то время как в случае мартенсита приближение среднего поля дает завышенные примерно на $100 \mathrm{~K}$ температуры $T_{C}{ }^{M}$.

Также можно отметить, что на фазовой диаграмме температуры Кюри аустенита $\left(T_{C}^{A}\right)$ уменьшаются, в то время как температуры Кюри мартенсита $\left(T_{C}{ }^{M}\right)$ увеличиваются с увеличением содержания Pt.

\section{4. Заключение}

В данной работе с помощью теории функционала плотности исследованы структурные и магнитные свойства для сплавов $\mathrm{Ni}_{2-x} \mathrm{Pt}_{x} \mathrm{MnGa}$. Геометрическая оптимизация аустенитной и устойчивость мартенситной фаз были выполнены с использованием пакета VASP в сочетании с подходом суперячейки из 16 атомов.
Было обнаружено, что равновесные параметры кристаллической решетки сплавов $\mathrm{Ni}_{2-x} \mathrm{Pt}_{x} \mathrm{MnGa}(0 \leq x \leq 2)$ увеличиваются с увеличением концентрации Pt. В общем, вычисленные значения параметра решетки находятся в хорошем согласии с экспериментальными данными. Вычисления полной энергии кристаллической решетки для искаженной структуры показали, что мартенситное состояние для $\mathrm{Ni}_{2} \mathrm{MnGa}$ может быть реализовано при соотношении $c / a=1,26$. Увеличение концентрации Pt приводит к увеличению сотношения $c / a=1,32$ (для $\mathrm{Ni}_{1,0} \mathrm{Pt}_{1,0} \mathrm{MnGa}$ ) и дальнейшему снижению до $c / a=1,30$ (для $\mathrm{Ni}_{0,25} \mathrm{Pt}_{1,75} \mathrm{MnGa}$ ). Используя данные параметров магнитного обменного взаимодействия, полученные с помощью пакета SPR-KKR, было показано, что наибольший вклад в полную энергию обменного взаимодействия связан с взаимодействием между парой ближайших соседних атомов Ni-Mn. B мартенситном состоянии значение данного взаимодействия увеличивается в 1,5-2 раза. Используя дальнодействующие обменные взаимодействия между магнитными атомами в классическом гамильтониане Гейзенберга, были определены температуры Кюри в рамках метода Монте-Карло и приближения среднего поля. Было показано, что температуры Кюри аустенита, полученные с помощью моделирования методом Монте-Карло и приближения среднего поля имеют близкие значения, в то время как в температуры Кюри мартенсита, полученные в рамках приближения среднего поля выше, чем температуры Кюри, полученные из моделирования с помощью метода Монте-Карло. Показано, что температура Кюри аустенита уменьшается, а температура Кюри мартенсита увеличивается с ростом концентрации $\mathrm{Pt}$ в сплавах $\mathrm{Ni}_{2-x} \mathrm{Pt}_{x} \mathrm{MnGa}$. Полученные в результате вычислений температуры Кюри находятся в хорошем согласии с экспериментальными данными. 
Благодарности/Acknowledgements. Работа выполнена при поддержке гранта РНФ № 17-72-20022-17 (раздел 3) и Правительства РФ (постановление №211 om 16.03.2013 2.)./This work was supported by the Russian Science Foundation grant no. 17-72-20022-17 (section 3), and the Government of the Russian Federation (ordinance no. 211 from 16.03.2013).

\section{Литература/References}

1. A. Sozinov, A. A. Likhachev, N. Lanska, K. Ullakko. Appl. Phys. Lett. 80, 1746 - 1748(2002). DOI: 10.1063/1.1458075

2. K. Ullakko, J. K. Huang, C. Kantner, and R. C. O'Handley, V. V. Kokorin. Appl. Phys. Lett. 69, $1966-1968$ (1996). DOI: $10.1063 / 1.117637$

3. S. J. Murray, M. Marioni, S. M. Allen, and R. C. O'Handley, T. A. Lograsso. Appl. Phys. Lett.77, 886 - 888 (2000). DOI: $10.1063 / 1.1306635$

4. V.D. Buchelnikov, V. V. Sokolovskiy. Phys. Met. Metallogr. 112, 633 - 665 (2011). DOI: 10.1134/S0031918X11070052

5. J. Pons, E. Cesari, C. Seguí, F. Masdeu, R. Santamarta. Mat.Sci. Eng. A. 481-482, 57-65 (2008). DOI: j.msea.2007.02.152

6. S. Singh, S.W. D'Souza, K. Mukherjee, P. Kushwaha, S. R. Barman, S. Agarwal, P.K. Mukhopadhyay, A. Chakrabarti, E. V. Sampathkumaran. Appl. Phys. Lett. 104, 231909 (2014). DOI: 10.1063/1.4883404
7. P. Entel, M. Siewert, M.E. Gruner, A. Chakrabarti, S.R. Barman, V.V. Sokolovskiy, V.D. Buchelnikov. J. Alloy. Compd. 577, S107-S112 (2013). DOI: j.jallcom.2012.03.005

8. S. Singh, S. W. D’Souza, J. Nayak, L. Caron, E. Suard, S. Chadov, C. Felser. Phys. Rev. B. 93, 134102 (2016). DOI: 10.1103/PhysRevB.93.134102

9. G. Kresse, J. Furthmuller. Phys. Rev. B. 54, 11169 (1996). DOI: 10.1103/PhysRevB.54.11169

10. G. Kresse, D. Joubert. Phys. Rev. B. 59, 1758 (1999) DOI: 10.1103/PhysRevB.59.1758

11. H. Ebert, D. Ködderitzsch, J. Minár. Rep. Prog. Phys. 74, 096501 (2011). DOI: 10.1088/0034-4885/74/9/096501

12. J. P. Perdew, K. Burke, M. Ernzerhof, Phys. Rev. Lett., 77, 18 (1996). DOI: 10.1103/PhysRevLett.77.3865

13. D. P. Landau and K. Binder. A Guide to Monte Carlo Simulations in Statistical Physics. Cambridge, Cambridge University Press. (2005) 432 p.

14. P.J. Webster, K.R. A. Ziebeck, S.L. Town, M.S. Peak. Philos. Mag. B. 49, 295-310 (1984). DOI: 10.1080/13642817408246515

15. V.V. Sokolovskiy, V.D. Buchelnikov, M.A. Zagrebin, P. Entel, S. Sahoo, M. Ogura. Phys. Rev. B. 86, 134418 (2012). DOI: 10.1103/PhysRevB.86.134418

16. V.A. Chernenko, V.A. Lvov, S.P. Zagorodnyuk, T. Takagi. Phys. Rev. B 67, 064407 (2003). DOI: 10.1103/ PhysRevB.67.064407 